\section{AB1001 ARE ANY DIFFERENCES IN THE LOCATION OF BONE LESIONS BETWEEN BONE MALIGNANT TUMORS AND INFLAMMATORY OSTEOPATHIES?}

Vyacheslav Silkov ${ }^{1,2}$, Nikita Suvorov², Mikhail Kostik², Olga Kopchak ${ }^{3}$, Alexei Maletin ${ }^{4}$, Vyacheslav Zorin ${ }^{4}$, Alexander Mushkin ${ }^{4} .{ }^{1}$ Saint-Petersburg clinical scientific and practical center for special types of medical care (oncology-oriented), Saint-Petersburg, Russian Federation; ${ }^{2}$ Saint-Petersburg State Pediatric Medical University, Saint-Petersburg, Russian Federation; ${ }^{3}$ Kirov's regional children's hospital, Kirov, Russian Federation; ${ }^{4}$ Science research Institute of Phthisiopulmonology, Saint-Petersburg, Russian Federation

Background: Different pediatric bone destructive diseases may have similar radiological findings but have their typical localizations [1].

Objectives: The aim of our study was to differences in the bone lesions location in various pediatric bone destructive diseases.

Methods: Our cohort $(n=204)$ consists of four main subtypes: i) chronic non-bacterial osteomyelitis ( $\mathrm{CNO}, \mathrm{n}=91$ ), ii) hematogenous osteomyelitis $(\mathrm{HO}, \mathrm{n}=47)$; iii) tuberculosis osteomyelitis (TBO, $n=33$ ), and iv) malignant bone tumors (MBT, $n=33$ ) - osteosarcoma and Ewing's sarcoma.

Results: Al bone destructive diseases have the similar frequency of femur and humerus involvement. MBT patients compare to others had the highest onset age, and higher frequency of pelvic involvent, and comparatively rare the involvement of spine, tibia, foot bones, sternum, the bones of forearm and hand (table).

Conclusion: Patients with bone destructive process with femur, humerus and pelvic involvement have to be under high level of consideration about all type of osteopathy, especially MBT.

This work supported by the Russian Foundation for Basic Research (grant № 18515-57001).

\section{REFERENCES}

[1] McCarville MB. The child with bone pain: malignancies and mimickers. Cancer Imaging. 2009;9 Spec No A:S115-21.

Disclosure of Interests: None declared DOI: 10.1136/annrheumdis-2019-eular.7898

\section{AB1002 IMMUNOGLOBULIN G4 RELATED DISEASE IN A 10 YEAR-OLD GIRL WITH MULTISYSTEM INVOLVEMENT}

Ovgu Kul Cınar ${ }^{1}$, Muthana Al-Obaidi ${ }^{2}$, Neil Sebire ${ }^{3}$, Parichat Khaosut ${ }^{4}$ Despina Eleftheriou ${ }^{5,6} .{ }^{1}$ Great Ormond Street Hospital NHS Foundation Trust, Paediatric Rheumatology, London, United Kingdom; ${ }^{1}$ Great Ormond Street Hospital NHS Foundation Trust, Paediatric Rheumatology, London, United Kingdom, ${ }^{3}$ UCL Great Ormond Street Institute of Child Health, Histopathology, London, United Kingdom; ${ }^{4}$ King Chulalongkorn Memorial Hospital, Paediatric Rheumatology, Bangkok, United Kingdom; ${ }^{5}$ UCL Institute of Child Health and Great Ormond Street Hospital NHS Foundation Trust, Paediatric and Adolescent Rheumatology, London, United Kingdom; ${ }^{6}$ ARUK Centre for Paediatric and Adolescent Rheumatology, Paediatric and Adolescent Rheumatology, London, United Kingdom

Background: IgG4-related disease (IgG4RD) is an immune-mediated fibroinflammatory condition characterized by the infiltration of IgG4-carrying plasma cells and storiform fibrosis in most of the tissues. The condition is reported to cause multisystem involvement, however salivary gland is the most commonly affected organ with IgG4-related sialadenitis. Raised IgG4 concentrations in the serum and prominent infiltration by plasmacytes expressing IgG4 in the lacrimal and salivary glands have been confirmed

Objectives: IgG4-RD has mostly been decribed in adult population and therefore generally not well-known among paediatricians. To the best of our knowledge, this patient is one of the rare paediatric cases in literature diagnosed with IgG4-RD. We intended to emphasize that IgG4RD should be kept in mind for differential diagnosis of the patients presenting with enlarged parotid, lacrimal and submandibular glands and sicca symptoms.

Methods: We extracted patient's clinical, laboratory and imaging data from our database and reviewed literature to reveal different manifestations of the IgG4RD.

Results: 10 year-old-girl presented with lacrimal and salivary gland swelling, sicca symptoms and fatigue. Ultrasound scan (USS) neck revealed multiple small lymph nodes and enlargement of both submandibular glands. Salivary glands also appeared bulky and heterogenous with multiple small hypoechoic focci. Appearances were likely to represent sialoadenitis and there was no convincing evidence of malignancy or Iymphoma. USS abdomen showed no abnormality. Full blood count, routine biochemistry and urine microscopy were normal. Autoantibodies came back as negative (ANA:Negative, ANCA: negative, Anti-Ro and Anti-La: negative, RF:negative, thyroid autoantibodies:negative). Anti-Cardiolipin IgG was weakly positive on the first visit, however repeated analyses were negative. IgG level was elevated in repeated samples. $\lg A$, $\lg M$ and $\lg E$ levels remained normal. IgG subgroups were performed which revealed significantly elevated IgG4 levels (21.49 Normal range: $0-1.1)$ IgG1, IgG2 and IgG3 levels were slightly elevated. The biopsy of salivary gland showed chronic inflammation with IgG4 staining and was suggestive of IgG4 related disorder. The patient was diagnosed with IgG4 related disease and treatment was started with intravenous methylprednisolone followed by Anti-CD20 (Rituximab) therapy and a weaning plan for steroids was given. Mycophenolate mofetil was commenced for the maintenance therapy. Patient has been in remission on maintenance therapy.

Conclusion: IgG4RD is a rare condition which can cause multisystem involvement with the infiltration of IgG4-bearing plasma cells in the tissues. We wanted to emphasize that this condition could also be seen in the paediatric population. Steroids are the cornerstone of the treatment, however Anti-CD20 medication (Rituximab) and steroid sparing agents such as mycophenolate mofetil could be the choice for maintenance therapy.

\section{REFERENCES}

[1] IgG4 related Disease: A disease Entity Developed in the 21st Century. Mimori T. Mod Rheumatol. 2019 Jan 24:1-2

[2] Major salivary gland enlargement in IgG4 related disease is associated with multiorgan involvement and higher basal disease activity. MartínNares E, Ángeles A, Hernandez-Molina G. Mod Rheumatol. 2019 Jan 24:1-15

Disclosure of Interests: None declared DOI: 10.1136/annrheumdis-2019-eular.2208

\begin{tabular}{|c|c|c|c|c|c|}
\hline Parameter & NBO & $\mathrm{HO}$ & TBO & MBT & $\mathrm{P}$ \\
\hline Onset age, years & $7.3(2.5-10.6)$ & $\begin{array}{c}10.9(6.2- \\
12.9)\end{array}$ & $\begin{array}{c}1.7(1.4- \\
3.4)\end{array}$ & $\begin{array}{c}12.0(10.0- \\
16.0)\end{array}$ & 0.00001 \\
\hline Number of foci, $\mathrm{n}(\%)$ & $3.0(1.0-6.0)$ & $1.0(1.0-1.0)$ & $\begin{array}{c}1.0(1.0- \\
1.0)\end{array}$ & $1.0(1.0-1.0)$ & 0.0001 \\
\hline Gender, males & $47(51.7)$ & $23(61.7)$ & $27(81.8)$ & $20(60.6)$ & 0.026 \\
\hline Monofocal involvement, $\mathrm{n}(\%)$ & $26(28.6)$ & $47(100.0)$ & $33(100.0)$ & $32(97.0)$ & 0.000001 \\
\hline Spine involvement, $\mathrm{n}(\%)$ & $29(31.9)$ & $2(4.3)$ & $1(3.0)$ & $1(3.0)$ & 0.000001 \\
\hline Femur involvement, n (\%) & $37(40.7)$ & $7(14.9)$ & $14(42.4)$ & $12(36.4)$ & 0.015 \\
\hline Tibia involvement, n (\%) & $33(36.3)$ & $12(25.5)$ & $6(18.2)$ & $4(12.2)$ & 0.029 \\
\hline Fibula involvement, n (\%) & $12(13.2)$ & $8(17.0)$ & $1(3.0)$ & $2(6.0)$ & 0.17 \\
\hline Foot involvement, $\mathrm{n}(\%)$ & $36(39.6)$ & $9(19.2)$ & $3(9.1)$ & $0(0.0)$ & 0.000005 \\
\hline Pelvis involvement, $n(\%)$ & $17(18.7)$ & $3(6.4)$ & $1(3.0)$ & $6(18.8)$ & 0.047 \\
\hline Sternum involvement, $n$ (\%) & $11(12.1)$ & $0(0.0)$ & & & \\
\hline Clavicula involvement, n (\%) & $10(11.0)$ & $1(2.1)$ & $0(0.0)$ & $1(3.0)$ & 0.045 \\
\hline Ribs involvement, $\mathrm{n}(\%)$ & $6(6.7)$ & $0(0.0)$ & $0(0.0)$ & $3(9.1)$ & 0.09 \\
\hline Humerus involvement, $\mathrm{n}(\%)$ & $10(11.0)$ & $4(8.5)$ & 7 (21.2) & $4(12.1)$ & 0.36 \\
\hline Maxilla involvement, $\mathrm{n}(\%)$ & $2(2.2)$ & $0(0.0)$ & $0(0.0)$ & $0(0.0)$ & 0.47 \\
\hline Radius involvement, $\mathrm{n}(\%)$ & $3(3.3)$ & $0(0.0)$ & $2(6.1)$ & $1(3.0)$ & 0.46 \\
\hline Ulna involvement, n (\%) & $3(3.3)$ & $1(2.1)$ & $2(6.1)$ & $0(0.0)$ & 0.52 \\
\hline Hand involvement, $\mathrm{n}(\%)$ & $4(4.4)$ & $1(2.1)$ & $0(0.0)$ & $0(0.0)$ & 0.37 \\
\hline Scapula involvement, n (\%) & $1(1.1)$ & $0(0.0)$ & $0(0.0)$ & $1(3.0)$ & 0.53 \\
\hline
\end{tabular}

